\title{
Recent Advances in the Local Drug Delivery for the Treatment of Restenosis
}

\author{
Pinak Khatri ${ }^{1,2 *}$ \\ ${ }^{1}$ Department of Product Development, G \& W PA Laboratories, USA \\ ${ }^{2}$ Department of Pharmaceutics, State University of New Jersey, USA \\ *Corresponding author: Pinak Khatri, Department of Product Development, Sellersville PA 18960, USA
}

Submission: 海 June 15, 2018; Published: 眥 July 13, 2018

\begin{abstract}
:
Stent procedure and balloon angioplasty are two major approaches used in the treatment of Cardio vascular diseases. There have been significant advances in the treatment of these disorders and complication resulting from these treatment options i.e. Restenosis. This article focuses on the evolution and the recent advances in the different treatment options available for the preventing Restenosis after vascular intervention. The focal point of this review is the approaches for a localized drug delivery in the vascular walls.
\end{abstract}

Keywords: Restenosis; Drug Delivery; Drug Eluting Stent; Drug Coated Balloon; Micro porous catheter

Abbreviations: LLL: Late lumen loss; BMS: Bare metal stent; DES: Drug Eluting stent; PAT: Paclitaxel; CVD: Cardio Vascular Diseases; NIH: Neo intimal Hyperplasia; TVR: Target Vessel Revascularization; SMC: Smooth Muscle Cell

\section{Introduction}

Around 80 million Americans suffer from at least one form of Cardio Vascular Diseases (CVD), which are the leading cause of death in the western world. Commonly practiced procedure to treat CVDs that leads to narrowing of blood vessels due to fatty plaque deposition includes balloon angioplasty and stenting [1]. The rates of acute and chronic vessel occlusion with "simple old balloon angioplasty" were unacceptably high at $30 \%$ to $60 \%$. The issue appears to eliminate by the usage of Bare-Metal Stents (BMS). However, it leads to the growth of new tissue, known as neointima, around and inter-twining the stent. This medical condition is called as Neointimal Hyperplasia (NIH) that subsequently leads to potentially devastating effects such as heart attack. Literature shows a strong and linear relationship between NIH formation and lumen loss. The Restenosis rates with BMS were reported to be between $16 \%$ and $44 \%$. Although the introduction of Drug Eluting Stent (DES) has reduced the incidence of Restenosis, they still continue to share the liability of increasing the associated risk of stent thrombosis as the bare metal stents. A conservative estimate suggests that the incidence of In-Stent Restenosis (ISR), requiring Target Vessel Revascularization (TVR), to be $5 \%$ to $10 \%$, with one estimate suggesting 200,000 repeat revascularizations in the US itself [2]. These techniques have evolved over the years to overcome complications arising out of the basic stent and balloon angioplasty procedures. This review focuses on the advances in local drug delivery and drug used in the treatment of Restenosis.

\section{Stent-based drug delivery}

Drug-eluting stents (DES): Few of the recent advances in the stent technology are changes in the material of construction, stent strut thickness, and development of application-specific dedicated stent platforms. There has been increasing use of titanium and a shift from the cobalt-chromium or stainless steel to platinumchromium alloys. The Axxess ${ }^{\mathrm{TM}}$, STENTYS DES and Cardio Mind Sparrow $^{\mathrm{TM}}$ (Biosensors) have a nitinol platform and are selfexpanding, while the TAXUS Petal and Nile PAX have a platinumchromium, and cobalt-chromium platform, respectively.

Another major advancement in stent design is the thickness of strut. Stent strut thickness is found to be associated with local inflammation at the site of the lesion. When it is excessive, it provides a formidable obstacle to neo-intimal stent-strut coverage. Therefore, strut thickness is crucial and studies have shown the relationship between strut thickness and rate of Restenosis. There was a positive correlation shown for Late Lumen Loss (LLL) with strut thickness in a meta-regression study. However, it should be noted that too much reduction in stent strut thickness might weaken the stent platform that can make it prone to longitudinal deformation, which is a problem that can only be partially offset by improvement in stent design [3].

Currently available DES dedicated to specific application like bifurcation lesions include Axxess ${ }^{\mathrm{TM}}$ containing Biolimus A9 
(Biosensors, CA, USA), the Paclitaxel-eluting Nile Pax® (Minvasys S.A.S., Gennevilliers, France), STENTYS (Stentys S.A.S., Paris, France) stents and TAXUS Petal (Boston Scientific) and for small vessels is Cardio Mind Sparrow ${ }^{\mathrm{TM}}$ (Biosensors) [4].

Drugs used in DES: Majority of Drugs used in preparing DES or given in conjugation with BMS are immunosuppressive, antiinflammatory and/or anti proliferative. Other drugs are also used to improve the endothelization rate and thereby restricting the chronic inflammation at the luminal side of the stent and for stent thrombosis. Drugs commonly used in the first generation stents included sirolimus and Paclitaxel, while later on zotarolimus and everolimus were used. Several clinical studies have confirmed the safety and efficacy of "limus family" and, especially, sirolimus has shown encouraging results in terms of LLL, binary Restenosis and late stent thrombosis. Actually, it still represents an ideal choice on the common final pathway of cell division cycle without excessive risks of inducing necrosis [3].

The newer drugs used in DES, mostly, from the -limus family include Biolimus A9 (umrolimus), novolimus and myolimus [4]. Biolimus A9 (umrolimus) is a highly Lipophilic (10 times more than its analogs) derivative of sirolimus, which is rapidly absorbed in tissues, and plays a role in reversibly inhibiting the growth factorstimulated cell proliferation [5]. It is utilized in Nobori® (Terumo, Tokyo, Japan) and Bio matrix Stents (Biosensors, Singapore). Newer anti-proliferating agents under investigation include cilostazol that is incorporated in the Cilotax Stent along with Paclitaxel. It is a potent inhibitor of phosphodiesterase and also has an anti-platelet effect. In CILOTAX trial, Cilotax and Paclitaxel dual DES was found to be associated with less in-stent thrombosis compared to Paclitaxel only DES [6]. In spite of the wide spread utility of DES, there have been some concerns related to late stent thrombosis, which may be associated with inflammatory response to stent coating and incomplete re-endothelization [7].

\section{Drug-eluting stent with Biodegradable Polymers}

In order to overcome the chronic inflammation of the vessel wall that is one of the commonly proposed mechanisms of stent thrombosis, DES with biodegradable polymers were developed. Biodegradable polymers like poly-l-lactide, poly lactic-co-glycolic acid, poly-d-lactide acid and their combinations have been used in DES. Synergy ${ }^{\mathrm{TM}}$ contains everolimus in a matrix of Poly lactic-coglycolic acid acting as a biodegradable polymer. There have been studies indicating the benefits of DES with biodegradable polymers [4]. DES containing biodegradable polymers was compared with the second generation DES containing permanent polymer in a meta-analysis and systemic literature review for randomized trials. The selected primary outcomes were definite stent thrombosis, myocardial infarction, target lesion revascularization, cardiac deaths and total deaths during the study period. The results showed no significant advantage for DES containing biodegradable polymers and the outcomes were comparable between both the groups [8].

\section{DES without polymer}

One way of avoiding the inflammatory response associated with the polymer is to develop a DES without polymer. Bio freedom $^{\text {TM }}$, containing Umirolimus (Biolimus A9), Yukon ${ }^{\circledR}$ Choice 4 and VESTA sync ${ }^{\mathrm{TM}}$ containing Sirolimus are a new generation of DES without polymer, where the drug is directly embedded into the metallic surface. The LEADERS FREE trial showed the superiority of the umirolimus-coated stent compared to Gazelle ${ }^{\mathrm{TM}}$ (Biosensors Interventional Technologies Pte Ltd., Singapore) with regard to TLR and MACE or stent thrombosis with only a month of utilization of DAPT [9]. The VESTA sync ${ }^{\mathrm{TM}}$ Eluting Stent (MIV Therapeutics, GA, USA) is made up of cobalt-chromium alloy with a $65 \mu \mathrm{m}$ strut thickness coated with a nanothin- micro porous hydroxyapatite surface coating impregnated with a low dose(55 $\mu \mathrm{g}$ ) of sirolimus without any polymer. The entire drug elutes within 60 days Comparison of VEST sync $\mathrm{c}^{\mathrm{TM}}$ and its bare metal stent version (without drug) showed that, at 8 months, the DES group had a late loss of $0.39 \pm 0.20 \mathrm{~mm}$ vs $0.78 \pm 0.60 \mathrm{~mm}$ compared to the bare metal version $(p=0.03)(4)$.

\section{Bio absorbable Stents}

There has been an interest in developing stents that not permanent i.e. bio absorbable stents. These stents are non-metallic scaffolds of biodegradable material like Poly-l-lactic acid that create a temporary mechanical support like metallic stents but degrade over time and hence eliminate the long-term risk associated with a metallic stent. It can also be used to deliver drugs locally. However, their presence beyond their intended functional period can result in various complications like chronic inflammation of the vessel wall, loss of vasomotion, in-stent atherosclerosis, restrictions to magnetic resonance imaging, difficulty with future vascularization and stent fracture [9]. The U.S. Food and Drug Administration approved the first fully bio absorbable stent-Absorb coronary stent in 2016 [10]. However, it was withdrawn from the market citing low commercial sales but there are proposed efforts to develop the second-generation of bio absorbable device [11].

There are also some bio absorbable scaffolds that have incorporated metallic alloy as bio absorbable scaffolds like AMS1 (Biotronik AG, Bülach, Switzerland). It contains WE43 alloy that contains $93 \%$ magnesium and $7 \%$ rare earth elements. It is similar to stainless steel stents in terms of mechanical properties like elastic recoil, collapse pressure and amount of shortening after inflation. Magnesium has also shown to possess anti-thrombogenic properties in vivo [12]. In a prospective, non-randomized, multicenter, clinical trial $(n=63)$ AMS-1 showed no safety concerns regarding deaths, myocardial infarction or scaffold thrombosis but showed lower long-term patency rates. Restenosis was shown to be due to faster than expected scaffold degradation leading to an early loss of radial force and resulting vessel coil. Therefore, to increase the retention time for the scaffold, AMS-1 was redesigned. The new design has higher collapse pressure than AMS-1 (1.5 vs. $0.8 \mathrm{bar}$ ). Also, the strut thickness was reduced from 165 to $120 \mu \mathrm{m}$. 
In order to reduce the neo intimal growth, the stent was coated with PLGA matrix containing Paclitaxel (DREAMS). Although there was a decrease in in-scaffold late lumen loss at 12 months $(0.52 \pm$ $0.39 \mathrm{~mm}$ ) compared to $1.08 \pm 0.49 \mathrm{~mm}$ of the AMS- 1 magnesium scaffold, the results did not match that of the currently available DES. Therefore, the scaffold was redesigned with 6-crown 2-link design and a strut thickness of $150 \mu \mathrm{m}$ and coated with a poly lactic acid matrix containing sirolimus(DREAMS $2 \mathrm{G}$ ) In the porcine coronary model, DREAMS $2 \mathrm{G}$ was found to be lower dismantling rate than DREAMS $1 \mathrm{G}$ [12].

Since stent has a sieve-like structure there may be heterogeneity in the drug distribution across the arterial walls with very high local drug concentration in the intimal layer adjacent to the stent struts while sub-minimal therapeutic concentration at intimal layer not adjacent to the struts. Approaches with uniform distribution of drug throughout the lesion site can prove be a superior pharmacotherapy.

\section{Balloon-based drug delivery}

There are two types of balloons used for drug delivery- DrugCoated Balloon (DCBs) and Drug Infused Balloon (DIBs). Ballooncatheter drug delivery technology is conceptualized based on a combination of conventional angioplasty and targeted drug delivery systems. Drug along with excipients are coated at the distal tip of the balloon catheter, which when inserted and inflated in the blood vessel at the target site, establishes a contact between the intimal wall of the blood vessel and the drug coating. The drug releases from the matrix to the local vascular lesion and maintains tissue concentration that can restrain the hyper proliferative response to vascular injury. Local delivery of drug using this platform allows for greater drug concentrations to be achieved at the site of vascular lesion, compared to systemic drug delivery [13].

However, the disadvantage of this approach includes loss of drug during balloon tracking, which results in the lower amount of drug delivered at the target site. In DIBs, the drug solution passes through the balloon and extrudes out of the porous balloon structure towards the lumen. Due to the poor solubility of Paclitaxel, cremophor is used to dissolve the drug; however, due to cremophor toxicity, amount of drug that can be delivered is limited. Moreover, the balloon inflation time of around three minutes is not adequate for effective drug uptake by the tissue. Therefore, efforts should be directed to improve the drug uptake by the tissue. In one of the studies, the nano particle albumin-bound PAT (nab-PAT) and cremophor-PAT were evaluated for an in vitro drug release, Smooth Muscle Cell (SMC) response, Endothelial Cell (EC) response, and in vivo drug uptake. It was observed that the nab-PAT was as effective as cremophor in inhibiting SMC growth while showing significantly greater amount of drug uptake [14]. The vascular endothelial cells, is a semi-selective permeability barrier, that controls the transport of fluids, electrolytes and macromolecules across the vessel wall [15].

The cell uptake of nab-PAT may be hindered by the larger molecular size of albumin and therefore, there is still some scope for further enhancing the cell uptake by using smaller molecules for conjugation with Paclitaxel. Since the local exposure time for the uptake of the drug is very less, any factor that can increase the endothelial cell permeability will be of utmost significance. Drugs can be modified physico chemically, while keeping the potency similar, or conjugated with a permeation enhancer to improve the penetration of the drug into the vascular tissue.

\section{Micro needle injection into adventitia:}

Delivery of drug to intima could potentially inhibit reendothelialization; however, delivery to the adventitia can reduce the exposure of the bulk drug to the intima that helps in minimizing systemic and local toxicity [16]. Therefore, delivery of drug to the adventitia instead of intima could be beneficial. An intraluminal micro infusion balloon catheter containing micro needles is introduced into the artery using a guide wire. Upon insufflations, the micro needles on the surface of the balloon pierce the vessel wall and deliver the drug in the adventitial layer. Lipophilic drugs would elute slower than a hydrophilic drug; thus forming a drugdepot from where the drug would slowly dissipate. Therefore, lower amounts of drug and shorter duration of drug delivery is required to achieve optimum local drug levels [17].

\section{Other approaches}

Other drug delivery approaches have also been explored that can be used in conjugation with stent procedure or balloon angioplasty. One of such approach is the use of magnetic nano particles containing drugs to target the lesion site. Dispersion of magnetic nano particles is injected systemically targeted using an external magnetic field to restrict the nano particles at the targeted site for greater drug uptake. Initial efforts using this technology did not achieve success in clinical studies due to the incapability to target deep tissue in human subjects as well as Un optimized and not fully biocompatible magnetic carrier formulations. However, efforts were made to optimize this technology to make it viable for enhanced efficiency and safety profiles [18].

\section{Conclusion}

There have been advances in both the major approaches for treatment of Restenosis-Drug eluting Stents and Drug Eluting Balloons. However, an effort to develop a targeted drug delivery with greater drug uptake is the current need to overcome the complications arising due to the use of Drug-Eluting Stents and Balloons. Targeting approach can be physical like magnet assisted targeting, chemical or biological. In order to improve the drug uptake, the drug can be Physico chemically modified to achieve greater permeation while retaining its potency. Moreover, permeation enhancers or conjugation with permeation enhancers can be explored to improve the drug uptake.

\section{Conflict of Interest}

None. 


\section{References}

1. Paul A, Shao W, Shum-Tim D, Prakash S (2012) The attenuation of Restenosis following arterial gene transfer using carbon nanotube coated stent incorporating TAT/DNAAng1 vegf nano particles. Biomaterials 33(30): 7655-7664.

2. Farooq V, Gogas BD, Serruys PW (2011) Restenosis: Delineating the numerous causes of drug-eluting stent Restenosis. Circ Cardiovasc Interv 4(2): 195-205

3. Lupi A, Schaffer A, Bongo AS (2018) Should ultrathin strut drug eluting stents be considered the new benchmark for novel coronary stents approval? the complex interplay between stent strut thickness, polymeric carriers and anti proliferative drugs. Journal of thoracic disease 10(2): 678-681

4. Costopoulos C, Latib A, Naganuma T, Sticchi A, Giannini F, et al. (2013) Newly available and recent advances in drug-eluting stents. Expert review of cardiovascular therapy 11(5): 555-566.

5. Seth A, Hiremath S, Dani S, Kapoor S, Jain R, et al. (2013) Clinical outcomes with bio limus (A9) ${ }^{\mathrm{TM}}$ eluting stent, 'Bio Matrix' In diabetic patients interim results from multicenter post market surveillance registry in India. Indian Heart J 65(5): 586-592.

6. Lee CW, Park DW, Seung KB, Kim PJ, Park HJ, et al. (2011) Comparison of dual drug-eluting Cilotax stent and Paclitaxel-eluting taxus liberte stent in native coronary artery lesions. Am J Cardiol 107(7): 990-994.

7. Virmani R, Farb A, Guagliumi G, Kolodgie FD (2004) Drug-eluting stents: Caution and concerns for long-term outcome. Coron Artery Dis Sep; 15(6): 313-318.

8. Pandya B, Gaddam S, Raza M, Asti D, Nalluri N, etal. (2016) Biodegradable polymer stents vs second generation drug eluting stents: A metaanalysis and systematic review of randomized controlled trials. World J Cardiol 8(2): 240-246.
9. Kalra A, Rehman H, Khera S, Thyagarajan B, Bhatt DL, et al. (2017) Newgeneration coronary stents: Current data and future directions. Curr Atheroscler Rep 19(4): 14.

10. http://www.fda.gov/MedicalDevices/ProductsandMedicalProcedures/ DeviceApprovalsandClearances/Recently-ApprovedDevices / ucm509951.htm.

11. https://www.dicardiology.com/article/abbott-will-end-sales-absorbbioresorbable-stent

12. Campos CM, Muramatsu T, Iqbal J, Zhang Y, Onuma Y (2013) Bio resorbable drug-eluting magnesium-alloy scaffold for treatment of coronary artery disease. International journal of molecular sciences 14(12): 24492-24500.

13. Tesfamariam B (2016) Local arterial wall drug delivery using balloon catheter system. J Controlled Release 238: 149-156.

14. Anderson JA, Remund T, Pohlson K, Lamichhane S, Evans C, et al. (2017) In vitro and in vivo evaluation of effect of excipients in local delivery of Paclitaxel using micro porous infusion balloon catheters. Journal of Biomedical Materials Research Part B: Applied Biomaterials 105(2): 376-390.

15. Fang J, Nakamura H, Maeda H (2011) The EPR effect: Unique features of tumor blood vessels for drug delivery, factors involved, and limitations and augmentation of the effect. Adv Drug Deliv Rev 63(3): 136-151.

16. Karanian JW, Peregoy JA, Chiesa OA, Murray TL, Ahn C (2010) Efficiency of drug delivery to the coronary arteries in swine is dependent on the route of administration: Assessment of luminal, intimal and adventitial coronary artery and venous delivery methods. Journal of Vascular and Interventional Radiology 21(10): 1555-1564.

17. Tesfamariam B (2017) Periadventitial local drug delivery to target Restenosis. Vascular pharmacology 1(17).

18. Chorny M, Fishbein I, Forbes S, Alferiev I (2011) Magnetic nano particles for targeted vascular delivery. IUBMB Life 63(8):613-620.

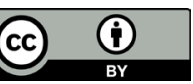

Creative Commons Attribution 4.0 International License

For possible submissions Click Here
Submit Article

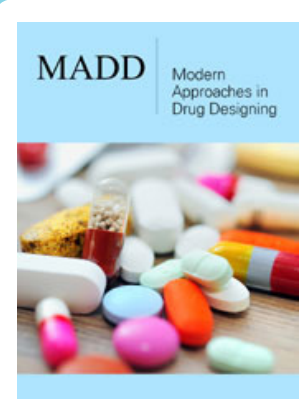

\section{Modern Approaches in Drug Designing \\ Benefits of Publishing with us}

- High-level peer review and editorial services

- Freely accessible online immediately upon publication

- Authors retain the copyright to their work

- Licensing it under a Creative Commons license

- Visibility through different online platforms 